\begin{tabular}{|c|l|}
\hline Title & $\begin{array}{l}\text { Three dimensional conformal fractionated radiotherapy for spinal schwannoma with a paravertebral or an intraosseous } \\
\text { component }\end{array}$ \\
\hline Author(s) & $\begin{array}{l}\text { Onimaru, Rikiya; Hida, Kazutoshi; Takeda, Naoki; Onodera, Shunsuke; Nishikawa, Y ukiko; Mori, Takashi; Shirato, } \\
\text { Hiroki }\end{array}$ \\
\hline Citation & $\begin{array}{l}\text { Japanese journal of radiology, 33(12), 757-763 } \\
\text { https://doi.org/10.1007/311604015-0492-4 }\end{array}$ \\
\hline Issue Date & $2015-12$ \\
\hline Doc URL & http://hdl.handle.net/2115/60829 \\
\hline Rights & ○ Japan Radiological Society 2015 \\
\hline Type & article \\
\hline File Information & s11604-015-0492-4.pdf \\
\hline
\end{tabular}

Instructions for use 


\title{
Three-dimensional conformal fractionated radiotherapy for spinal schwannoma with a paravertebral or an intraosseous component
}

\author{
Rikiya Onimaru ${ }^{1} \cdot$ Kazutoshi Hida $^{2} \cdot$ Naoki Takeda $^{3} \cdot$ Shunsuke Onodera $^{1} \cdot$ \\ Yukiko Nishikawa $^{1} \cdot$ Takashi Mori $^{1} \cdot$ Hiroki Shirato $^{1}$
}

Received: 25 August 2015 / Accepted: 9 October 2015 / Published online: 27 October 2015

(C) Japan Radiological Society 2015

\begin{abstract}
Introduction We retrospectively evaluated the efficacy of three-dimensional conformal radiotherapy (3D-CRT) for spinal schwannoma.

Methods Nine patients with spinal schwannoma were treated with 3D-CRT. All patients had a paravertebral or intraosseous component. Tumor sizes ranged from 0.8 to $8.7 \mathrm{~cm}$, with a median of $3.5 \mathrm{~cm}$. The prescribed dose was $50 \mathrm{~Gy}$ in 25 fractions at the isocenter, except for 1 patient who received $66 \mathrm{~Gy}$ in 33 fractions for a large sacral tumor. The follow-up period ranged from 20 to 137 months, with a median of 72 months.

Results Tumor shrinkage within $3 \mathrm{~mm}$ occurred in 4 patients and tumor expansion within $3 \mathrm{~mm}$ occurred in 3 . One tumor showed neither expansion nor shrinkage at the last follow-up. One patient experienced transient expansion by $8 \mathrm{~mm}$ in diameter at 12 months after the completion of radiotherapy (35-43 $\mathrm{mm}$ ), and then the tumor size remained unchanged for 7 years. No severe late toxicity $\geq$ grade 3 was observed.

Conclusions Only 1 of 9 tumors showed transit expansion over $3 \mathrm{~mm}$ after 3D-CRT, and severe late radiation toxicity was not observed. Use of 3D-CRT should be considered a treatment option for spinal schwannoma.
\end{abstract}

Rikiya Onimaru

ronimaru@pop.med.hokudai.ac.jp

1 Department of Radiation Medicine, Hokkaido University Graduate School of Medicine, North 15 West 7, Kita-ku, Sapporo 060-8638, Japan

2 Department of Neurosurgery, Hokkaido University Graduate School of Medicine, Sapporo, Japan

3 Department of Rehabilitation Science, Faculty of Health Science, Hokkaido University, Sapporo, Japan
Keywords Spinal schwannoma Paravertebral schwannoma $\cdot$ Three-dimensional conformal radiotherapy $(3 \mathrm{D}-\mathrm{CRT}) \cdot$ Fractionated radiotherapy $\cdot$ Conventional fractionation

\section{Introduction}

Spinal schwannoma is generally treated by surgery [1-3]. Conti et al. reported the results of 174 total removals out of 179 spinal neurinomas [1]. Their total removal rate is high. The recovery rate in Klekamp-Samii's scoring system was about $76 \%$, and the symptoms encountered before surgery improved in about $17 \%$. Seppala et al. reported that the life expectancy of the patients with spinal schwannoma is comparable to that of the general population after surgery [2].

Although total removal of spinal schwannoma has shown excellent results, it is sometimes difficult to completely remove such tumors. Seppala et al. reported that 20 of 187 schwannoma lesions were treated with partial or subtotal removal in order to avoid injury to a major root or the spinal cord [2]. In addition, a late complication may occur after surgery. Seppala et al. reported the following rates of late complications: cystic myelopathy in $2 \%$ of the patients, spinal arachnoiditis in $6 \%$, spinal deformity in $6 \%$, and troublesome pain in $7 \%$ [2]. In a study of dumbbell-type schwannoma, Satoh et al. reported that 3 of 4 patients showed postoperative neurological deficits and 1 of the 3 patients never recovered [3].

Radiosurgery for a spinal tumor is recognized as a therapeutic option for patients for whom surgery is difficult or inadvisable [4-6]. Radiosurgery for spinal schwannomas was reported as effective [4-6], but despite the efficacy of radiosurgery for spinal schwannoma, there are concerns about whether the linear-quadratic model is reliable enough 
at a high dose per fraction [7], and the evidence regarding spinal tolerance doses after radiosurgery is still sparse $[8$, 9].

Vestibular schwannoma is treated by radiosurgery or fractionated radiotherapy $[10,11]$, and the results seem to be comparative between these two methods $[10,12]$. Aoyama et al. reported that only 10 of 200 patients with vestibular schwannoma underwent surgery because of tumor expansion after fractionated radiotherapy [12]. Varughese et al. reported that the 5-year retreatment-free survival rate of patients who underwent radiosurgery was $93.9 \%$ in a prospective setting [10]. It is likely that fractionated radiotherapy for spinal schwannoma will show excellent clinical results, as it has for vestibular schwannoma. However, there are few reports about fractionated radiotherapy for spinal schwannoma [13].

We have used 3-dimensional conformal radiotherapy (3D-CRT) with conventional fractionation (of which the dose per fractionation was 2 Gy) since July 2000 to treat patients with spinal schwannoma at our institute, because we considered that conventional fractionation was safer than radiosurgery because of a significant amount of evidence about the long-term tolerance of organs at risk (OAR) irradiated with conventional fractionation. Here, we retrospectively reviewed our data and evaluated the efficacy of 3D-CRT with conventional fractionation for spinal schwannoma.

\section{Methods}

Nine patients with spinal schwannoma were treated at Hokkaido University Hospital between 2000 and 2008 with conventional fractionation with a fraction size of 2 Gy. None of the patients had neurofibromatosis. Their ages ranged from 11 to 77 years old (median, 52 years).
The tumor sizes ranged from 0.8 to $8.7 \mathrm{~cm}$ (median, $3.2 \mathrm{~cm}$ ). Five patients were male and four female. One tumor was located at the cervical spine, 3 at the thoracic spine, 4 the lumbar spine, and 1 at the sacrum. All patients had a paravertebral or intraosseous component. Six patients had an intracanal and 1 an intraforaminal component. Six patients had a dumbbell-type tumor. The initial symptom was pain for 7 patients, muscle weakness for 2, and neurogenic bladder for 1; 1 patient experienced no symptoms. Four patients underwent surgery before radiotherapy; 3 of the 4 underwent partial removal and postoperative radiotherapy was performed. The maximum tumor size of these 3 tumors before surgery ranged from 4.0 to $6.8 \mathrm{~cm}$. All 3 patients had pain, and 1 had gait disturbance. After surgery, all patients showed improvement of pain, and 1 patient with gait disturbance showed improvement of symptoms. The other patient experienced recurrence 2 years after surgery, and salvage radiotherapy was delivered for the recurrent tumor. The size of this tumor before surgery was not available in the chart. This patient suffered from back and bilateral leg pain and could not walk alone. These symptoms were improved after partial removal of the intracanal tumor, and this patient was able to walk with a cane. One patient had 3 lesions; 1 of the 3 was treated by surgery alone, another was treated by radiotherapy alone, and the other lesion was observed and not treated. The lesion treated by radiotherapy is included in this study and the lesion treated by surgery alone excluded. Table 1 shows all of the patient characteristics.

Treatment planning was made based on the CT findings. The CT slice thickness ranged from 1 to $5 \mathrm{~mm}$. All patients received 3D CRT. The radiotherapy planning system (RTP system) used was FOCUS [Focus, Computerized Medical Systems (CMS), St. Louis, MO] for six patients and $\mathrm{XiO}(\mathrm{CMS})$ for three. The homogeneity

Table 1 Patient characteristics

\begin{tabular}{|c|c|c|c|c|c|c|c|c|c|c|}
\hline Case & Age & Gender & Tumor location & Axial location & Dumbbell type & $\begin{array}{l}\text { MTD* before surgery } \\
(\mathrm{cm})\end{array}$ & $\operatorname{MTD}^{*}(\mathrm{~cm})$ & TD (Gy) & Policy of RT & Marker \\
\hline 1 & 57 & Female & Rt L3/4 & Parav & No & - & 0.8 & 50 & D & Yes \\
\hline 2 & 60 & Male & Lt Th3/4 & Parav & No & - & 3 & 50 & D & Yes \\
\hline 3 & 63 & Male & Lt L1/2 & Isc and parav & Yes & - & 3.2 & 50 & $\mathrm{D}$ & Yes \\
\hline 4 & 15 & Female & Rt C2-C3 & Isc and parav & Yes & 4 & 3 & 50 & $\mathrm{P}$ & Yes \\
\hline 5 & 77 & Female & Rt L4-S1 & Isc and parav & Yes & NA & 8.7 & 66 & S & No \\
\hline 6 & 11 & Male & Lt Th3/4 & Isc and parav & Yes & 4.2 & 3.5 & 50 & $\mathrm{P}$ & Yes \\
\hline 7 & 51 & Male & Lt Th12/L1 & If and parav & No & - & 2 & 50 & $\mathrm{D}$ & Yes \\
\hline 8 & 52 & Male & Lt L5/S1 & Isc and parav & Yes & - & 5.4 & 50 & $\mathrm{D}$ & No \\
\hline 9 & 51 & Female & sacrum & Isc and ios & Yes & 6.8 & 4.9 & 50 & $\mathrm{P}$ & No \\
\hline
\end{tabular}

$M T D$ maximum tumor diameter before radiotherapy, $T D$ total dose, parav paravertebral, isc intraspinal canal, if intraforaminal, ios intraosseous, $D$ definitive, $P$ postoperative, $S$ salvage, $R T$ radiotherapy 
correction method was Clarkson for seven patients and superposition for two. All patients were treated with a linear accelerator (EXL-20DP; Mitsubishi Electronics, Tokyo) with multileaf collimators (leaf width, $5 \mathrm{~mm}$ ). The X-ray energy was $10 \mathrm{MV}$, except for 1 patient treated with 4-MV X-ray for cervical schwannoma. The total prescribed dose was 50 Gy in 25 fractions at the isocenter, except for 1 patient who received 66 Gy in 33 fractions for a large sacral tumor (maximum diameter $8.7 \mathrm{~cm}$ ). A gold marker was implanted in 6 of the patients in order to reduce the planning target volume (PTV) using a realtime tracking radiotherapy system (RTRT system) with a previously reported method [13]. Markers were implanted percutaneously into the paraspinal muscle near the tumor under local anesthesia in 3 patients and by surgical implantation to the vertebral body in 3 . The reasons why a marker was not inserted in the other 3 patients were postoperative recurrence after initial surgery, surgery at another hospital, and the physicians' decision, respectively. We did not have any IGRT method other than the RTRT system during the study period so that IGRT could not be used for these 3 patients. The RTRT system was used for the image-guided radiotherapy (IGRT) technique for 6 patients with fiducial markers, but other methods such as cone beam CT were not used for other patients.

The clinical target volume was the same as the gross tumor volume. The margin for the PTV was 3-5 $\mathrm{mm}$ when a marker was implanted; otherwise, a 10-20-mm margin was added. The number of ports ranged from 2 to 10 , with a median of 3 . The field-in-field technique was used in two patients. Non-coplanar beam arrangement was used in 5 patients. The maximum dose to the spinal cord was set not to exceed $50 \mathrm{~Gy}$. The maximum spinal cord dose of the patient who received 66 Gy was under 50 Gy because the distance between the tumor and conus medullaris was far enough to avoid irradiation to the conus medullaris. Overall treatment time (OTT) ranged from 38 to 60 days, with a median of 43 days. Figure 1 is an example of the dose distribution of which the prescribed dose was $50 \mathrm{~Gy}$ in 25 fractions at the isocenter using the field-in-field technique.

Local progression was defined as an increase larger than $3 \mathrm{~mm}$ in the maximum tumor diameter compared to the same measurement at the pretreatment evaluation. Based on the report that the average growth rate of the vestibular schwannoma was $3.87 \mathrm{~mm} /$ year when observation was chosen for the initial policy [11], we considered that an increase larger than $3 \mathrm{~mm}$ was appropriate for the definition of local progression. The local control period was calculated from the last day of radiotherapy delivery. Local control was judged using charts, imaging including MRI or CT, and reports of diagnostic imaging. The followup period ranged from 20 to 137 months, with a median of 72 months. Late radiation toxicity was evaluated using

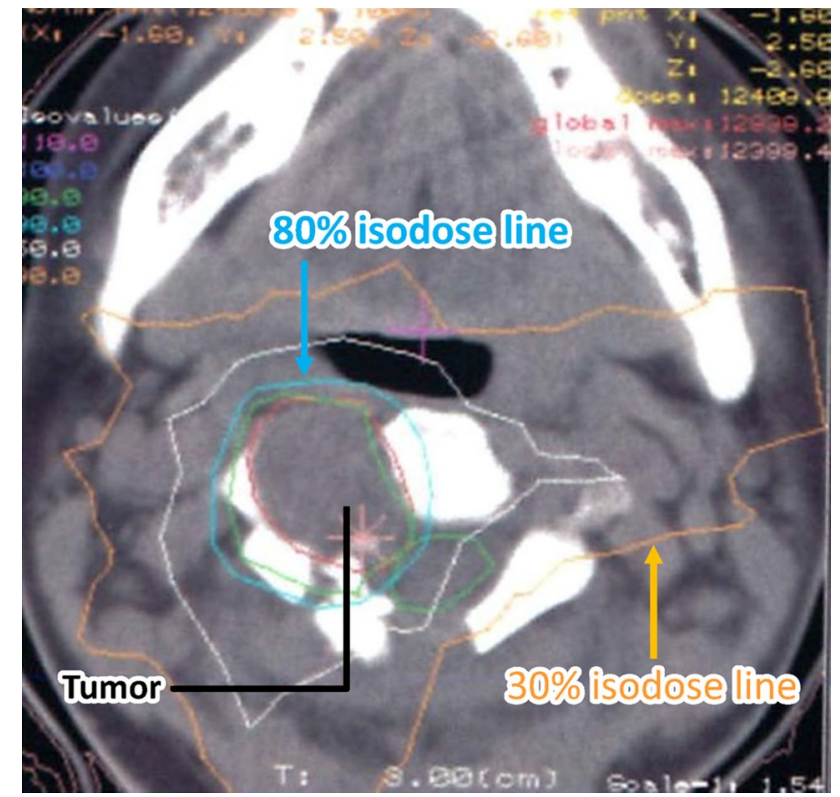

Fig. 1 Dose distribution of cervical schwannoma treated with 50 Gy in 25 fractions. Field-in-field technique was used to reduce the dose to the spinal cord. White arrows indicate the $80 \%$ (i.e., 40 Gy in 25 fractions) isodose line, and black arrows indicate the $30 \%$ (i.e., 15 Gy in 25 fractions) isodose line

the NCI Common Terminology Criteria for Adverse Events (CTCAE), version 4.0 .

\section{Results}

Tumor shrinkage occurred in four patients, and tumor expansion within $3 \mathrm{~mm}$ had occurred in three patients at the last follow-up. One tumor showed neither expansion nor shrinkage at the last follow-up. Tumor progression occurred in 1 patient. The patient experienced transit expansion ( $8 \mathrm{~mm}$ in diameter, from 35 to $43 \mathrm{~mm}$ ) 12 months after completing radiotherapy, and then the tumor size did not change for about 7 years. Figure 2 shows the dose distribution and MRI at 94 months after radiotherapy.

Three of the seven patients who suffered from pain experienced pain relief. The other four patients with pain showed no change in pain. Other symptoms showed neither improvement nor deterioration. One patient who had no symptoms before radiotherapy felt intermittent leg pain 5 months after the completion of radiotherapy. This patient mentioned at 19 months' follow-up that the leg pain occurred three times per year. The maximum spinal cord dose of this patient was 4651 cGy in 25 fractions; the dose distribution of this patient is shown in Fig. 3. Table 2 summarizes these results.

No severe late toxicity $\geq$ grade 3 in CTCAE v 4.0 was observed. 
Fig. 2 a Dose distribution and MRI T1-weighted image of the patient whose tumor expanded transiently. b The MRI image was obtained at 94 months after radiotherapy. The tumor expanded 12 months after the radiotherapy's completion, and its size remained stable for about 7 years
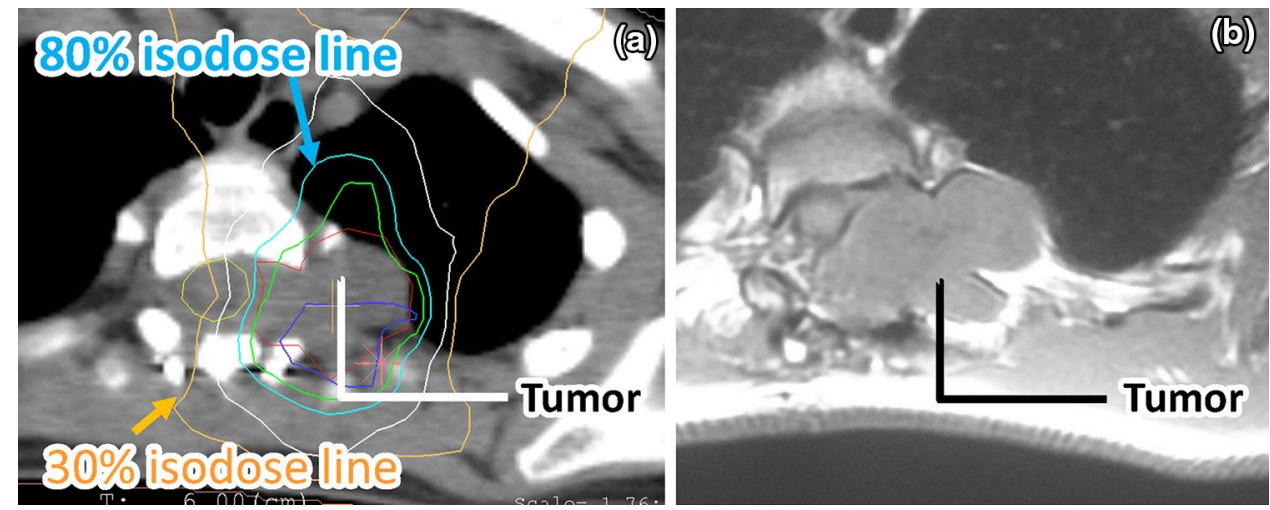

Fig. 3 Dose distribution of the patient who had no symptoms before radiotherapy but felt intermittent leg pain after radiotherapy. The maximum spinal cord dose was 4651 cGy in 25 fractions
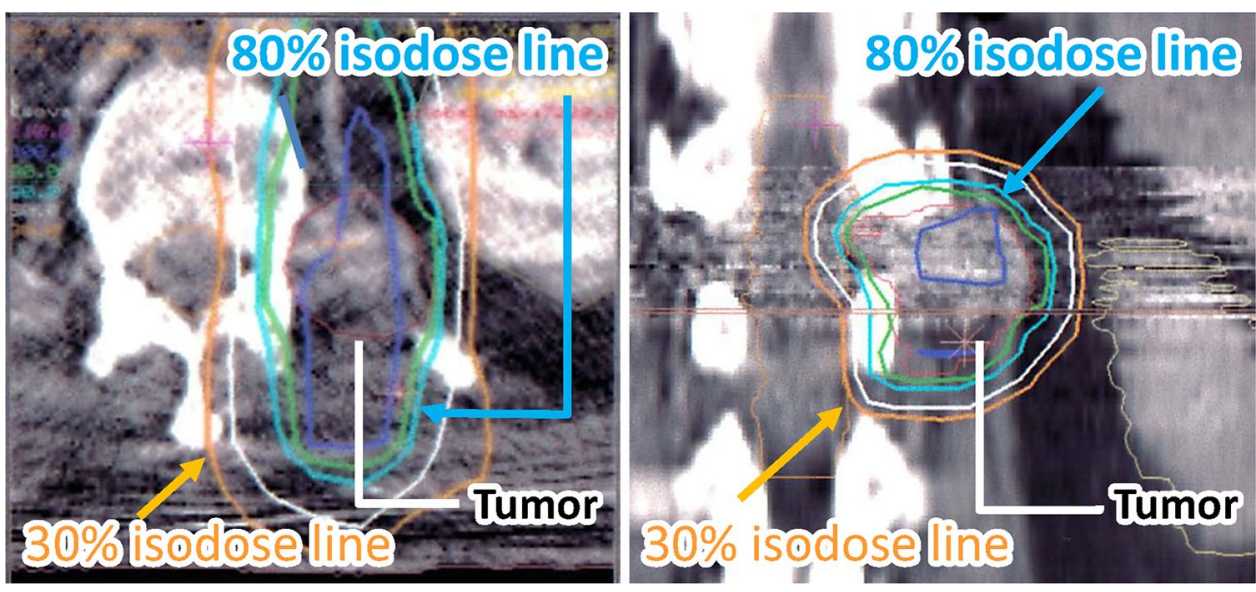

Table 2 Summary of results of radiotherapy

\begin{tabular}{|c|c|c|c|c|c|c|}
\hline Case & MTD before RT $(\mathrm{cm})$ & $\begin{array}{l}\text { MTD at last follow-up } \\
(\mathrm{cm})\end{array}$ & Initial symptom & $\begin{array}{l}\text { Improvement of } \\
\text { symptom after surgery }\end{array}$ & $\begin{array}{l}\text { Improvement of } \\
\text { symptom after RT }\end{array}$ & $\begin{array}{l}\text { Follow-up period } \\
\text { (year) }\end{array}$ \\
\hline 1 & 0.8 & 0.5 & Pain, MW & - & I (pain), NC (MW) & 6 \\
\hline 2 & 3 & 3 & Pain & - & $\mathrm{NC}$ & 11.4 \\
\hline 3 & 3.2 & 3.4 & Pain & - & I & 1.7 \\
\hline 4 & 3 & 2.7 & Pain & I & $\mathrm{I}$ & 4.7 \\
\hline 5 & 8.7 & 8.6 & Pain & I & $\mathrm{NC}$ & 8.9 \\
\hline 6 & 3.5 & 4.3 & MW & I & $\mathrm{NC}$ & 7.8 \\
\hline 7 & 2 & 1.8 & None & - & $\mathrm{D}$ & 6.7 \\
\hline 8 & 5.4 & 5.6 & Pain & - & $\mathrm{NC}$ & 5 \\
\hline 9 & 4.9 & 5 & Pain, NB & I & $\mathrm{NC}$ & 4.4 \\
\hline
\end{tabular}

$M T D$ maximum tumor diameter, $M W$ muscle weakness, $N B$ neurogenic bladder, $R T$ radiotherapy, $I$ improvement, $N C$ no change, $D$ deterioration

\section{Discussion}

In this study, nine patients with spinal schwannoma who were treated by conventional fractionated radiotherapy showed no severe late radiation toxicity, and the changes in size were within $3 \mathrm{~mm}$ in 8 of 9 tumors. One patient experienced a transient expansion of the tumor, but the tumor size then remained stable for approximately 7 years.
Considering that there are few reports about conventional fractionated radiotherapy for spinal schwannoma, the present findings are valuable, although the study was retrospective and the number of patients small.

Surgery for paravertebral spinal schwannoma is sometimes invasive, and thus radiotherapy may be indicated for paravertebral schwannoma. Surgery for paravertebral schwannoma requires a facetectomy that may causes 
spinal instability without spinal fusion. In order to prevent the instability, posterior spinal fusion with many vertebrae is required. In addtion, a cost-transversectomy or thoracotomy is sometimes required for a thoracic paravertebral schwannoma to completely remove the tumor. These procedures are extremely invasive and not suitable for patients with mild symptoms. The present study showed that fractionated radiotherapy is less invasive and often suitable for patients with a thoracic paravertebral spinal schwannoma.

There are few reports about fractionated radiotherapy for spinal schwannoma [13], but there are several reports about radiosurgery for spinal schwannoma. Radiosurgery for spinal schwannoma has been reported as effective [4-6]. Selch et al. reported the clinical results of image-guided linear accelerator-based radiosurgery for 25 nerve sheath tumors [4]. They observed no local failure after radiosurgery with a median peripheral dose of 12 Gy and a median followup period of 18 months. Sachdev et al. reported that $51 \%$ of 47 schwannomas were stable in size and $47 \%$ showed tumor shrinkage [5]. The average prescribed dose was $18.74 \mathrm{~Gy}$, and the average maximum dose was $23.75 \mathrm{~Gy}$. Gerszten et al. reported that only 3 of 35 patients with spinal schwannomas underwent surgery after radiosurgery with a mean prescribed maximum dose of 22.03 Gy because 2 patients showed significant numbness and the other had a large $\mathrm{C} 1$ tumor [6].

Although radiosurgery for spinal schwannoma shows excellent results, fractionated radiotherapy for spinal schwannoma has several favorable points. There is a useful amount of evidence concerning late radiation reactions to conventional fractionation radiotherapy [14]. The possibility of a late radiation reaction after treatment for benign disease should be carefully monitored because of the long life expectancy of patients. The life expectancy of patients with spinal schwannoma can be expected to be similar to that of the general population, according to Seppala et al. [2].

Late radiation reactions after conventional radiotherapy may be seen in the esophagus, blood vessels, intestine, and colon, as well as the spinal cord. Cox et al. reported that serious esophageal toxicity (grade 4 or above) occurred after paraspinal radiosurgery in 5 out of 204 spinal metastases [15]. The late radiation reaction of these OARs after fractionated radiotherapy for spinal schwannoma can be more precisely predicted based on a great deal of evidence about conventional radiotherapy for other diseases than that of radiosurgery for spinal schwannoma.

Our study shows spinal schwannoma can be treated with 50 Gy in 25 fractions, which is under the spinal cord tolerance dose [14]. These characteristics make conventional fractionated radiotherapy for spinal schwannoma safe. On the other hand, the maximum dose of 13 Gy to the spinal cord in a single fraction with radiosurgery is thought to be safe [16], and Selch et al. reported no spinal cord toxicity with a median peripheral dose of 12 Gy [4]. Contrary to their suggestions, Gerszten et al. reported that 2 of 35 patients with schwannoma experienced spinal cord toxicity after radiosurgery even though the volume of the spinal cord above 8 Gy was $<0.02 \mathrm{~cm}^{3}$ [6]. The two patients were treated with a tumor marginal dose of $20 \mathrm{~Gy}$ in a single fraction. Vestibular schwannoma is well controlled with a peripheral dose of $12 \mathrm{~Gy}$ [10], and the marginal dose of 20 Gy seems to be higher than the optimal dose [6]. Because $50 \mathrm{~Gy}$ in 25 fractions is under the tolerance dose of the spinal cord, it is unlikely that a patient who receives this dose will experience radiation myelopathy after conventional fractionated radiotherapy, even if a setup error occurs or is larger than expected.

For tumors larger than $10 \mathrm{~cm}$, radiotherapy should be done with a linear accelerator, because the field sizes of radiosurgery-dedicated equipment are generally less than $10 \times 10 \mathrm{~cm}$. Since there may be the risk of spinal cord compression due to transit tumor expansion, especially in large tumors, the combination of surgery for intra-canal tumors and fractionated radiotherapy for extra-canal tumors should be considered.

However, fractionated radiotherapy has unfavorable points compared with radiosurgery. First, the OTT of the fractionated radiotherapy for spinal schwannomas is apparently longer than that of radiosurgery. In our study, the OTT ranged from 38 to 60 days. This long OTT may be unfavorable for some patients.

Second, the potential for patient setup error in fractionated radiotherapy without IGRT may be larger than that in radiosurgery. We used IGRT with a real-time tracking system for six patients with gold fiducial markers in this study as reported [13], but not for the other three patients. Ryu et al. reported that isocenter accuracy was within $2 \mathrm{~mm}$ using the Novalis system (Brain-LAB, Munich, Germany) [17], and Furweger et al. reported that the mean target error was within $1 \mathrm{~mm}$ using the CyberKnife image-guided radiosurgery system (Accuray, Sunnyvale, CA) [18]. If fractionated radiotherapy is performed without IGRT, the precise patient set-up is difficult. However, a relatively large setup error in fractionated radiotherapy might not result in a serious problem. Because the prescribed dose of fractionated radiotherapy for spinal schwannoma is under the tolerance dose of the spinal cord, esophagus and peripheral nerves, we are able to treat patients safely with fractionated radiotherapy with a relatively large setup margin without IGRT, although we completely agree that a precise setup is preferable, especially for benign tumors.

We treated three patients with a combination of surgery and radiotherapy. It is known that transient expansion may occur after radiotherapy for vestibular schwannomas. Aoyama et al. reported that $21 \%$ of patients with vestibular 
schwannoma treated with fractionated radiotherapy experienced tumor expansion, although $69 \%$ of the expanded tumors shrank after the expansion [12]. We experienced one patient whose tumor showed expansion by about $8 \mathrm{~mm}$. It is possible that tumor expansion in the spinal canal results in spinal cord compression. In order to avoid spinal compression after radiotherapy, it seems reasonable to use surgery as the initial treatment to remove the tumor within the spinal canal followed by radiotherapy to treat any tumor outside of the extra-vertebral foramen. Because it is sometimes impossible to preserve both of the affected ventral nerve roots and the dorsal root $[3,19]$, surgery for a dumbbell-shaped schwannoma is difficult, and the frequency of adverse events is higher than that for surgery of tumors within the spinal canal [3]. The combination of surgery and radiotherapy is an option for the treatment of dumbbell type schwannomas, especially for tumors with a large extradural component.

Patients who are not candidates for surgery are indicated for radiotherapy. Recurrence after surgery, medical comorbidities such as cardiovascular disease, and multiple tumors are appropriate indications for radiotherapy. We treated patients with recurrence or multiple lesions. Since local control can be excellent after conventional fractionated radiotherapy, this treatment should be considered as an alternative to surgery when surgery is not indicated.

\section{Conclusions}

In conclusion, only 1 of 9 tumors showed transit expansion over $3 \mathrm{~mm}$, and 3 of 7 patients who suffered from pain experienced pain relief after fractionated conventional radiotherapy. Severe late radiation toxicity was not observed. Although radiosurgery for spinal schwannoma shows excellent results, fractionated radiotherapy should be considered as a treatment option in light of the significant amount of evidence regarding normal-tissue late radiation toxicity and the ability of fractionated radiotherapy to treat large tumors safely, especially combined with surgery for intracanal tumors.

\section{Compliance with ethical standards}

Funding This work was supported in part by a Grant-in-Aid for Science Research from the Ministry of Education, Science, Sports and Culture, Japan.

Ethical statement The Institutional Review Board at Hokkaido University Hospital approved this retrospective study in December 2012.

Conflict of interest Hiroki Shirato received research findings from Shimadzu Corp., Hitachi Medico, and Hitachi Co., Ltd.

\section{References}

1. Conti P, Pansini G, Mouchaty H, Capuano C, Conti R. Spinal neurinomas: retrospective analysis and long-term outcome of 179 consecutively operated cases and review of the literature. Surg Neurol. 2004;61(1):34-43 (discussion 4).

2. Seppala MT, Haltia MJ, Sankila RJ, Jaaskelainen JE, Heiskanen O. Long-term outcome after removal of spinal schwannoma: a clinicopathological study of 187 cases. J Neurosurg. 1995;83(4):621-6. doi:10.3171/jns.1995.83.4.0621.

3. Satoh N, Ueda Y, Koizumi M, Takeshima T, Iida J, Shigematsu K, et al. Assessment of pure single nerve root resection in the treatment of spinal schwannoma: focus on solitary spinal schwannomas located below the thoracolumbar junction. J Orthop Sci. 2011;16(2):148-55. doi:10.1007/s00776-011-0032-8.

4. Selch MT, Lin K, Agazaryan N, Tenn S, Gorgulho A, DeMarco $\mathrm{JJ}$, et al. Initial clinical experience with image-guided linear accelerator-based spinal radiosurgery for treatment of benign nerve sheath tumors. Surg Neurol. 2009;72(6):668-74. doi:10.1016/j.surneu.2009.04.019 (discussion 74-5).

5. Sachdev S, Dodd RL, Chang SD, Soltys SG, Adler JR, Luxton $\mathrm{G}$, et al. Stereotactic radiosurgery yields long-term control for benign intradural, extramedullary spinal tumors. Neurosurgery. 2011;69(3):533-9. doi:10.1227/NEU.0b013e318218db23 (discussion 9).

6. Gerszten PC, Burton SA, Ozhasoglu C, McCue KJ, Quinn AE. Radiosurgery for benign intradural spinal tumors. Neurosurgery. 2008;62(4):887-95. doi:10.1227/01.neu.0000318174.28461.fc (discussion 95-6).

7. Kirkpatrick JP, Meyer JJ, Marks LB. The linear-quadratic model is inappropriate to model high dose per fraction effects in radiosurgery. Semin Radiat Oncol. 2008;18(4):240-3. doi:10.1016/j. semradonc.2008.04.005.

8. Medin PM, Boike TP. Spinal cord tolerance in the age of spinal radiosurgery: lessons from preclinical studies. Int $\mathbf{J}$ Radiat Oncol Biol Phys. 2011;79(5):1302-9. doi:10.1016/j. ijrobp.2010.10.052.

9. Ryu S, Jin JY, Jin R, Rock J, Ajlouni M, Movsas B, et al. Partial volume tolerance of the spinal cord and complications of singledose radiosurgery. Cancer. 2007;109(3):628-36. doi:10.1002/ cncr.22442.

10. Varughese JK, Wentzel-Larsen T, Pedersen PH, Mahesparan R, Lund-Johansen M. Gamma knife treatment of growing vestibular schwannoma in norway: a prospective study. Int $\mathrm{J}$ Radiat Oncol Biol Phys. 2012;84(2):e161-6. doi:10.1016/j.ijrobp.2012.03.047.

11. Shirato H, Sakamoto T, Sawamura Y, Kagei K, Isu T, Kato T, et al. Comparison between observation policy and fractionated stereotactic radiotherapy (SRT) as an initial management for vestibular schwannoma. Int J Radiat Oncol Biol Phys. 1999;44(3):545-50.

12. Aoyama H, Onodera S, Takeichi N, Onimaru R, Terasaka S, Sawamura Y, et al. Symptomatic outcomes in relation to tumor expansion after fractionated stereotactic radiation therapy for vestibular schwannomas: single-institutional long-term experience. Int J Radiat Oncol Biol Phys. 2013;85(2):329-34. doi:10.1016/j.ijrobp.2012.05.003.

13. Onimaru R, Shirato H, Aoyama H, Kitakura K, Seki T, Hida K, et al. Calculation of rotational setup error using the real-time tracking radiation therapy (RTRT) system and its application to the treatment of spinal schwannoma. Int J Radiat Oncol Biol Phys. 2002;54(3):939-47.

14. Emami B, Lyman J, Brown A, Coia L, Goitein M, Munzenrider JE, et al. Tolerance of normal tissue to therapeutic irradiation. Int J Radiat Oncol Biol Phys. 1991;21(1):109-22. 
15. Cox BW, Jackson A, Hunt M, Bilsky M, Yamada Y. Esophageal toxicity from high-dose, single-fraction paraspinal stereotactic radiosurgery. Int J Radiat Oncol Biol Phys. 2012;83(5):e661-7. doi:10.1016/j.ijrobp.2012.01.080.

16. Kirkpatrick JP, van der Kogel AJ, Schultheiss TE. Radiation dose-volume effects in the spinal cord. Int $\mathrm{J}$ Radiat Oncol Biol Phys. 2010;76(3 Suppl):S42-9. doi:10.1016/j. ijrobp.2009.04.095.

17. Ryu S, Fang Yin F, Rock J, Zhu J, Chu A, Kagan E, et al. Imageguided and intensity-modulated radiosurgery for patients with spinal metastasis. Cancer. 2003;97(8):2013-8. doi:10.1002/ cncr.11296.
18. Furweger C, Drexler C, Kufeld M, Muacevic A, Wowra B, Schlaefer A. Patient motion and targeting accuracy in robotic spinal radiosurgery: 260 single-fraction fiducial-free cases. Int J Radiat Oncol Biol Phys. 2010;78(3):937-45. doi:10.1016/j. ijrobp.2009.11.030.

19. Jiang L, Lv Y, Liu XG, Ma QJ, Wei F, Dang GT, Liu ZJ. Results of surgical treatment of cervical dumbbell tumors: surgical approach and development of an anatomic classification system. Spine. 2009;34(12):1307-14. 